\title{
Modelo teórico para levantamento e organização de subsunçores no âmbito da Aprendizagem Significativa
}

Theoretical model for surveying and organizing subsumers within the scope of Meaningful Learning

\author{
Olavo Leopoldino da Silva Filho ${ }^{1 @}$, Marcello Ferreira ${ }^{* 1}$ \\ ${ }^{1}$ Universidade de Brasília, Instituto de Física, Brasília, DF, Brasil.
}

Recebido em 20 de setembro de 2021. Revisado em 23 de dezembro de 2021. Aceito em 21 de janeiro de 2022.

The Meaningful Learning Theory presents two absolutely crucial steps to reach the goal of meaningful teaching. The survey of subsumers, followed by their advanced organization, are conditions of possibility for learning to break the barrier of a merely mechanical learning, aimed at ephemeral memorization. However, such steps are very often disregarded, even by those who explicitly assume this theoretical framework. The survey stage of subsumers, in particular, is riddled with subjectivities on the part of those who organize learning, which brings the extra difficulty of being able to establish comparisons between surveys of subsumers by different teachers. The advanced organization stage, in turn, suggests a personalized teaching approach, since students' cognitive structures can differ, and usually do, widely. This work seeks to present a methodology that tackles both problems. Without neglecting the inherent subjectivity of the survey of subsumers, it presents a formalization of it, through the fuzzy inference technique, also presenting its computational implementation. The issue of personalization of teaching based on this methodology is also addressed.

Keywords: Meaningful Learning Theory. Subsumers Investigation. Advanced Organizers. Fuzzy Thinking. Theoretical Model.

\begin{abstract}
A Teoria da Aprendizagem Significativa apresenta duas etapas absolutamente cruciais para que se atinja o objetivo de se ensinar significativamente. O levantamento de subsunçores, seguido de sua organização avançada, são condições de possibilidade para que a aprendizagem possa romper a barreira de uma ocorrência meramente mecânica, voltada para memorizações efêmeras, atingindo níveis sofisticados de (re)significação cognitiva. Entretanto, tais etapas são muito frequentemente desconsideradas, mesmo por aqueles que assumem, explicitamente, esse referencial teórico. O levantamento de subsunçores, em particular, se mostra eivado de subjetividades da parte de quem organiza a aprendizagem, o que traz a dificuldade extra de se poder estabelecer comparações entre levantamentos de subsunçores por diferentes professores. A etapa de organização avançada, por sua vez, sugere uma abordagem de ensino personalizado, visto que as estruturas cognitivas dos estudantes podem diferir, e normalmente o fazem, largamente. Este trabalho busca, portanto, apresentar uma metodologia que ataca ambos problemas. Sem descurar da subjetividade inerente do levantamento de subsunçores, apresenta um consentâneo modelo teórico de formalização, por meio da técnica de inferências fuzzy, apresentando, ainda, sua implementação computacional. Aborda-se, ainda, a questão da personalização do ensino a partir dessa metodologia.
\end{abstract}

Palavras-chave: Teoria da Aprendizagem Significativa. Levantamento de Subsunçores. Organização de Subsunçores. Inferências fuzzy. Modelo teórico.

\section{Introdução}

David Ausubel [1, 2] é considerado o idealizador da Teoria da Aprendizagem Significativa (TAS), tendo contado com notórios desdobramentos de seus colaboradores, como os relativos aos domínios educacional, integrativo de pensamentos, sentimentos e ações [36], e epistemológico, articulando professor, estudante e materiais na construção de significados [7]. A partir de sua publicação, esse corpo teórico tem sido apresentado e desenvolvido em todo o mundo e, em especial, no Brasil 8 20, país em que recebeu aceitação particularmente favorável em decorrência da participação

\footnotetext{
*Endereço de correspondência: marcellof@unb.br
}

de pesquisadores e professores universitários em pósgraduações orientadas por Ausubel e seus colaboradores, certamente influenciados por um alinhamento político e cultural aos modelos educacionais norte-americanos. Desde então, a TAS tem recebido importantes aportes e extensões que a têm tornado um importante referencial para a didática nos níveis básico e superior, nas diversas áreas do conhecimento e, com ainda mais impacto, nas ciências da natureza, em virtude do caráter abstrato representável, modelável e esquematizável - e da estrutura hierarquizada do corpo de conhecimentos [21, 22].

Como abordagem psicológica e de caráter cognitivista, a TAS tem como conceito central aquele de estrutura cognitiva, entendida como um conjunto organizado de ideias de dado indivíduo, seja em determinado assunto, 
seja em um campo específico do conhecimento [22. Por seu caráter cognitivista, a TAS privilegia as noções de aquisição, armazenamento e organização das ideias na constituição da estrutura cognitiva, consignando que sua cúspide é a capacidade de acionamento, uso e ressignificação de conhecimentos em problemáticas contextualizadas e avançadas.

Nesse sentido, a aprendizagem é considerada como um processo de ampliação e reorganização da estrutura cognitiva frente a elementos que nela não se encontram ou encontram-se em desordem, desconexos. Entretanto, essa ampliação pela assimilação de novos conceitos pode ocorrer de maneiras distintas, sempre na relação que estabelecem com aqueles já existentes na estrutura cognitiva. Assim, uma aprendizagem significativa, conceitodiretor da TAS, só ocorre quando a assimilação se estabelece de maneira não arbitrária, isto é, quando os novos conceitos são assimilados pela estrutura cognitiva a partir de uma ancoragem conceitual em outros já existentes.

Os conceitos que possibilitam essa forma substantiva e não arbitrária de assimilação, e que são cientificamente consentidos, são os chamados subsunçores 23. Eles constituem, mesmo que por amorfia, conhecimentos prévios especificamente relevantes relativamente ao tema em questão para a aprendizagem de outros conhecimentos, que não se reduzem apenas à sua expressão em conceitos individuais, mas também se podem representar por conexões entre conceitos na forma proposicional.

Do que foi dito, fica clara a centralidade da noção de subsunçores na TAS. De fato, a acepção de aprendizagem significativa é dela tributária. A busca pelo levantamento dos subsunçores, relevantes a dado assunto, presentes na estrutura cognitiva dos estudantes, aos quais supostamente se deseja ensinar algo, é uma etapa essencial do processo de ensino e aprendizagem - de fato, pode-se afirmar que é uma das condições de possibilidade para a ocorrência de aprendizagem significativa.

A TAS, evidentemente, se organiza para muito além do levantamento dos subsunçores. De fato, nessa abordagem, outra etapa essencial do processo de ensino e aprendizagem é aquela em que se faz a organização avançada (também traduzida como organização prévia) dos subsunçores encontrados nas estruturas cognitivas dos estudantes. Essa etapa é particularmente importante para que os subsunçores encontrados possam ser organizados com vistas àquilo que se deseja efetivamente ensinar, uma vez que podem se encontrar desorganizados, esparsos, ou fragilmente fixados na estrutura cognitiva do aprendiz.

A despeito de sua óbvia importância no aparato lógico e epistemológico da TAS, as duas etapas previamente mencionadas, de levantamento dos subsunçores e de organização avançada destes, seguem recebendo menor atenção dos estudiosos da aprendizagem significativa, e, principalmente, daqueles que a usam como parte do seu referencial teórico [21, 22].
Em pesquisas que utilizam a TAS como parte do seu referencial teórico, o levantamento de subsunçores é feito, usualmente, aplicando-se um teste, com questões abertas ou fechadas, cabendo ao professor inferir, a partir de tais testes, quais subsunçores estão ou não presentes na estrutura cognitiva dos estudantes. Tal inferência não raro se mostra eivada de equívocos, em particular por ser guiada excessiva e não sistematicamente pela subjetividade do professor. O problema, ressalta-se, não é o da subjetividade envolvida, de caráter irredutível, como se verá, mas de seu caráter assistemático. Com isso, por exemplo, torna-se praticamente impossível comparar o levantamento de subsunçores de dois professores distintos, feitos para um mesmo assunto em condições homogêneas, ou mesmo aperfeiçoar um mesmo levantamento de maneira sistemática. O levantamento de subsunçores, em geral, rompe a barreira do subjetivo para recair no espaço do idiossincrático.

A etapa da organização avançada, evidentemente, sofre com tais dificuldades, uma vez que se constrói a partir do que se verifica na etapa de levantamento de subsunçores. Mais ainda, ela aponta para uma abordagem de ensino por personalização [24, uma vez que as estruturas cognitivas dos estudantes irão, quase sempre, diferir consideravelmente. Isso traz, por sua vez, o problema de se trabalhar com um ensino personalizado em turmas com grande número de estudantes, o que certamente pode ser mitigado, mas não absolutamente resolvido, por meio do uso das tecnologias digitais de informação e comunicação (TDIC). Isso é particularmente viável na cultura digital que ambienta (ou, ao menos, deveria ambientar) a educação contemporânea, marcada pela hiperconectividade, pelas aprendizagens em rede e por inteligências coletivas, disruptivas e flexíveis, traço cognitivo apropriadamente orgânico à TAS [25]31.

Em face dessas contingências, mas também das possibilidades que as perspectivas da aprendizagem significativa numa cultura digital trazem à qualificação da didática, lato sensu, e da didática em física, stricto sensu, esta investigação tem por objetivo apresentar um modelo teórico e uma respectiva técnica de levantamento de subsunçores, consistente com a TAS e seus requisitos, que ordenem objetivamente os elementos subjetivos irredutíveis do processo, permitindo eventuais comparações, para um mesmo tema, de diferentes aplicações, assim como a possibilidade de estender e/ou aperfeiçoar o levantamento de subsunçores de maneira sistemática.

Associada a essa técnica, este trabalho também sugere abordagens no processo de organização avançada dos subsunçores levantados, subentendendo, pois, toda a etapa preparatória que subjaz a qualquer processo de aprendizagem significativa. Como resultado desta abordagem, vislumbra-se a possibilidade de se fazer o levantamento dos subsunçores, assim como sua organização prévia, de maneira automática, envolvendo as TDIC, em particular as disponíveis na WEB, oferecendo aportes 
para o planejamento e desenvolvimento de processos personalizados e flexíveis de ensino.

\section{O levantamento de subsunçores}

Na década de 1970, Joseph Novak, trabalhando no interior de uma abordagem cognitivista, introduziu a noção de mapas conceituais como forma de organizar e representar o conhecimento [6, 32,34. Essa ideia, que rapidamente foi absorvida pela TAS, é ferramenta importante no contexto desta teoria e essencial para a presente discussão.

Mapas conceituais podem ser formalmente concebidos como grafos orientados em que comparecem um conjunto de nós (representando os conceitos) e arestas (representando conexões predicativas entre tais conceitos) 35. Assim, uma estrutura predicativa da forma $P_{a}\left(n_{1}, n_{2}\right)$ estabelece a conexão entre os nós n1 e n2 por um vínculo predicativo $P_{a}$. Os nós $n_{1}$ e $n_{2}$ podem ser, eles mesmos, estruturas predicativas (e.g. proposições), gerando uma recursão potencialmente infinita capaz de assegurar as complexidades de conexões usuais de campos semânticos. Nesse sentido, um mapa conceitual (ou grafo) é, formalmente um modelo do tipo $\left\langle\left\{n_{i}\right\},\left\{P_{a}\left(n_{i}, n_{j}\right)\right\}\right\rangle$, consistindo em um conjunto de nós e um conjunto concreto de nós conectados formando estruturas proposicionais. Na Figura 1 apresenta-se um exemplo genérico de mapa conceitual dado por

$$
\left\langle\left\{n_{1}, \ldots, n_{6},\right\},\left\{P_{1}\left(n_{1}, n_{2}\right), \ldots, P_{6}\left(n_{1}, n_{2}\right),\right\}\right\rangle
$$

Com base nessa representação, a técnica que se propõe para o processo de levantamento dos subsunçores deve se estruturar e organizar com base nos passos que se seguem, a priori tomados como hipóteses teóricas cuja viabilização metodológica e a implementação computacional serão demonstradas à frente:

1. Criar um mapa conceitual relativo aos conceitos e conexões que se deseja ensinar (conceitosalvo: $\left.C A=\left\langle\left\{n_{i}\right\},\left\{P_{a}\left(n_{i}, n_{j}\right)\right\}\right\rangle\right)$. Nesse mapa,

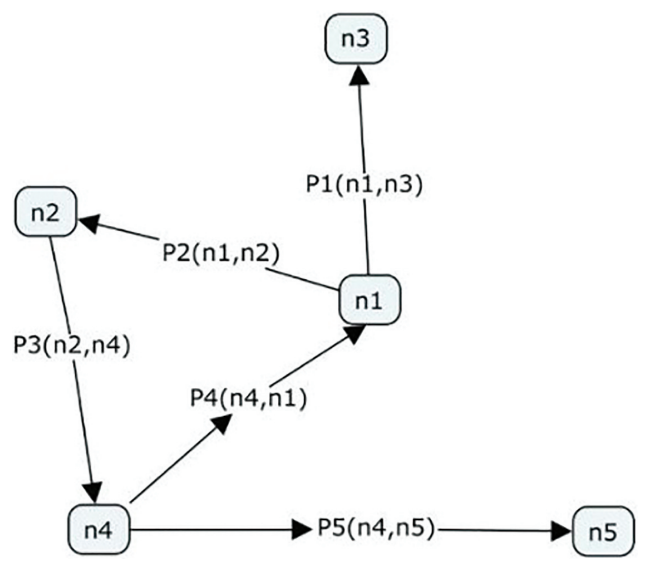

Figura 1: Exemplo genérico de mapa conceitual. Fonte: elaboração própria (2021). devem constar apenas os conceitos que se deseja efetivamente ensinar, devendo ser o mais conciso possível.

2. Fazer um mapa conceitual $C S=\left\langle\left\{s_{i}\right\},\left\{Q_{a}\right.\right.$ $\left.\left.\left(s_{i}, s_{j}\right)\right\}\right\rangle$ apenas com os conceitos que se julgam necessários para se ensinar CA. Este mapa apresentará os conceitos e conexões que devem, idealmente, estar presentes na estrutura cognitiva de cada estudante para que o aprendizado almejado tenha boas chances de ser bem-sucedido. Decorrerá desse mapa a ação de levantamento dos subsunçores.

3. Os conceitos e conexões devem apresentar, frente ao que se deseja ensinar, graus diferentes de relevância $\rho$ no intervalo $[0,1]$ - note o caráter subjetivo e difuso dessa ação, visto que se está falando em graus de relevância (difusividade) a serem atribuídas pelo professor (subjetividade) aos itens. Assim, para cada nó e para cada conexão efetiva entre os nós, cada qual representado em um item individual, atribui-se um grau de relevância no intervalo $[0,1]$. Os itens relativos aos nós representam levantamento de subsunçores de conhecimento substantivo ou definicional, enquanto que os itens relativos às arestas do grafo representam conhecimento proposicional.

4. Os itens do questionário podem ser abertos ou fechados (múltipla escolha), mas, para as pretensões deste trabalho, serão adotados apenas itens da segunda categoria.

(a) Itens fechados (preferíveis) devem acessar cada um dos termos e conexões individualmente (daí a importância de o mapa conceitual ser suscinto). Itens abertos, de modo geral, acessarão mais de um elemento, seja mais de um termo, seja mais de uma conexão.

(b) Cada alternativa deve ter uma classificação para o grau de importância com que contribui para o estabelecimento daquele subsunçor:

i. Se os itens são fechados com resposta única, esta representação será um dos elementos do conjunto $\{0,1\}$;

ii. Se os itens são fechados de resposta múltipla, deve-se atribuir a cada alternativa distintos graus de importância, de modo que as representações variem no intervalo: $[-1,1]$. Os conceitos que recebem escores negativos representam aquelas alternativas que, se marcadas, implicam confusão ou concepção equivocada. Por sua vez, os conceitos que recebem escores positivos representam conhecimentos que, se marcados, evidenciam compreensão efetiva. Independentemente do intervalo da escala, sugere-se que os escores das alternativas, para um mesmo item, sejam de soma total nula. 
iii. Se os itens forem abertos, os conceitos e/ou conexões que eles acessam devem estar listados (para o professor, com base na estrutura conceitual da matéria de ensino) e, a partir das respostas dos estudantes, devem ser atribuídos graus de importância a cada um desses conceitos e/ou conexões.

Como o interesse originário é desenvolver uma metodologia que permita a análise e a tomada de decisão automática acerca da subsunção, por um computador, a partir dos resultados obtidos, adotaremos a estratégia (ii) acima.

$\mathrm{Na}$ Figura 2, são apresentados exemplos de construção de perguntas do tipo (ii), buscando-se simplificar as representações de relevância e de importância (para detalhes, ver a seção seguinte).

Uma vez que o instrumento de avaliação tenha sido respondido pelos estudantes, passa-se à etapa de organização avançada, que deve usar um algoritmo que otimize a apresentação de materiais digitais adicionais, justamente como forma de organização dos subsunçores. Tal processo deve apresentar materiais adicionais para:

- Alternativas consideradas mais importantes para a compreensão do subsunçor relacionado ao item, considerando-se a relevância do item (do subsunçor), mas que não foram marcadas pelo estudante; ou

- Alternativas consideradas de importância alta (mas apresentando erros conceituais) para a compreensão do subsunçor relacionado ao item, e que foram marcadas pelo estudante.

Na seção que se segue, apresenta-se, em linhas gerais, o processo de organização avançada.

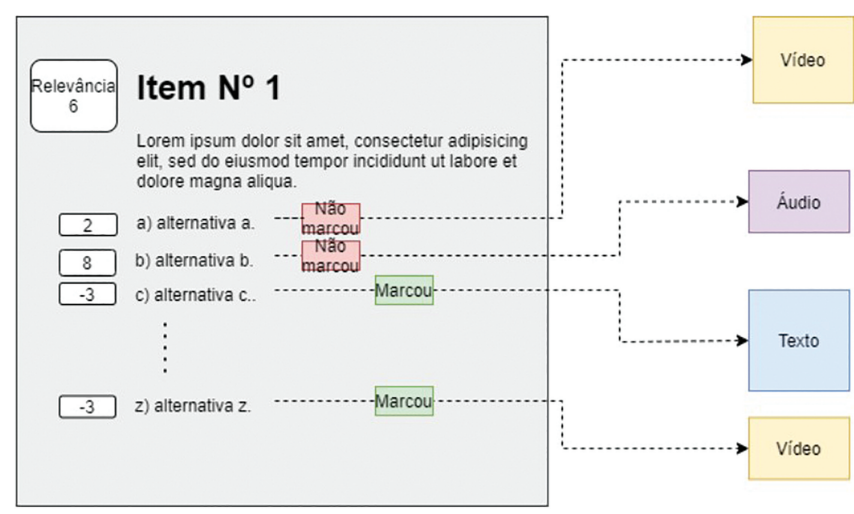

Figura 2: Construções das perguntas que servirão de levantamento de subsunçores, com representação do processo de organização avançada, que pode ser feito também automaticamente pela apresentação de vídeos, textos, áudios, dentre outros. Fonte: elaboração própria (2021).
2.1. Características subjetivas e difusas na construção de um algoritmo de levantamento de subsunçores

- Graus de relevância do item: input feito pelo professor.

- Graus de importância de cada alternativa: input feito pelo professor.

- Marcações de cada alternativa: input feito pelo estudante.

Esses parâmetros são os inputs para o programa que irá realizar o procedimento de levantamento dos subsunçores e produzir elementos para sua organização automática.

As atribuições de relevância e de importância são, evidentemente, subjetivas e, além do mais, difusas conceitos cujas fronteiras (ou "margens") não são bem definidas. Ocorre que não é por serem subjetivas que são livres para qualquer atribuição; espera-se que os professores concordem (subjetividade) aproximadamente (difusividade) com as marcações uns dos outros, de modo que a metodologia que se desenvolve neste trabalho pretende, justamente, servir como medium para este tipo de diálogo.

Da mesma maneira, o processo de inferência usado para passar das atribuições de relevância às de necessidade de organização dos subsunçores, para cada estudante, a despeito de depender dos resultados (objetivos) das respectivas marcações, é igualmente subjetivo, pela maneira como se deseja sopesar esses graus de relevância.

Assim, um algoritmo voltado para o tratamento automatizado do processo de organização avançada precisa implementar um processo que emule os processos psicológicos que estão associados às inferências que um especialista (um professor) poderia fazer. Como as variáveis de item e de alternativa são claramente difusas, isto é, não possuem clivagens precisas, mas fronteiras cinzentas, uma solução que se impõe imediatamente é a do uso de sistemas de inferência difusa [36]. A variável principal, neste caso, é a de presença do subsunçor na estrutura cognitiva, que será modelada a partir da noção de conjuntos difusos. Na seção seguinte, descrevese, brevemente, o funcionamento da lógica difusa e das inferências que ela possibilita.

\section{Lógica e pensamento difuso e estratégias de decisão: bases para a construção de um sistema de inferência para o levantamento de subsunçores}

Nesta seção, apresenta-se esquematicamente apenas os elementos principais da modelagem difusa de sistemas, deixando ao leitor interessado a sugestão de consultar [36], ou bibliografia equivalente [37], para mais detalhes. 
É o próprio [37, p. 3], a propósito, que registra que

\begin{abstract}
A Teoria de Conjuntos Fuzzy e os Conceitos de Lógica Fuzzy podem ser utilizados para traduzir em termos matemáticos a informação imprecisa expressa por um conjunto de regras linguísticas. Se um operador humano for capaz de articular sua estratégia de ação como um conjunto de regras da forma se ... então, um algoritmo passível de ser implementado em computador pode ser construído.
\end{abstract}

O conceito fundamental na modelagem difusa (do tipo que aqui interessa) é a de função de pertinência de um conjunto. Para conjuntos difusos, a relação de pertinência $\chi$ a um conjunto $A$ é uma função que toma elementos de um conjunto universo $U$ e atribui a eles um valor $v$ no intervalo $[0,1]$, isto é,

$$
\begin{gathered}
\chi[A]: U \rightarrow[0,1] \\
v \in U \rightarrow 0 \leq \chi[A](v) \leq 1
\end{gathered}
$$

Um conjunto difuso é o par $(A ; \chi[A])$, que é uma relação (e pode ser contínuo ou discreto). Assim, um conjunto difuso (discreto) construído a partir do conjunto $A=\{1,2,3\}$, poderia ser, por exemplo,

$$
A_{D}=\{(1 ; 0,2),(2 ; 0,5),(3 ; 0,8)\},
$$

que diz que $1 \in A$ com 'intensidade' 0,$2 ; 2 \in A$ com 'intensidade' 0,5 ; e $3 \in A$ com 'intensidade' 0, 8. Se o conjunto $A$ é contínuo, então $A_{D}$, evidentemente, tornase uma função. Por paralelismo, uma variável linguística é de tal sorte que seus valores são nomes de conjuntos difusos.

Com as definições anteriores, pode-se imediatamente generalizar as operações acerca de conjuntos difusos como extensões imediatas das operações entre conjuntos usuais.

Um conjunto difuso se diz normal se existe algum elemento $v$ para o qual $\chi[A](v)=1$ (é importante ter todos os conjuntos difusos normalizados). Os elementos $v \in U$ para os quais $\chi[A](v)>0$ formam o suporte de $A_{D}$, enquanto que os elementos para os quais $\chi[A](v)=$ 1 , formam o cume de $A_{D}$. $\mathrm{O}$ conjunto universo $U$ pode ser escrito como $\chi[U](x)=1$ e o conjunto vazio, como $\chi[\emptyset](x)=0$.

A partir dessas definições, tem-se as seguintes relações:

- De inclusão:

$$
A_{D} \subset B_{D} \Longleftrightarrow(\forall v \in U)[\chi[A](v) \leq \chi[B](v)] .
$$

- De igualdade:

$$
A_{D}=B_{D} \Longleftrightarrow\left\{A_{D} \subset B_{D} \wedge B_{D} \subset A_{D}\right.
$$

que é equivalente a

$$
(\forall x \in U)[\chi[A](v)=\chi[B](v)] .
$$

- De união:

$$
\begin{aligned}
A_{D} \cup B_{D} & =\{(v, \chi[A \cup B]) \mid \chi[A \cup B](v) \\
& =\max (\chi[A](v) ; \chi[B](v))\},
\end{aligned}
$$

em que max( ) é a função máximo ${ }^{1}$

- De interseção:

$$
A_{D} \cap B_{D}=\{\chi[A \cap B](v)=\min (\chi[A](v) ; \chi[B](v))\},
$$

em que min( ) é a função mínimo.

- De complemento:

$$
{\overline{\left(A_{D}\right)}}_{B_{D}}=\{(v, \chi[\bar{A}] \mid \chi[\bar{A}])=\max (0 ; \chi[A]-\chi[B])\},
$$

em que

$$
{\overline{\left(A_{D}\right)}}_{B_{D}},
$$

representa o complemento de $A$ em $B$ difuso.

- De complemento/negação:

$$
\bar{A}_{U}=\neg A_{D}=\{(v, \chi[\bar{A}] \mid \chi[\bar{A}])=1-\chi[A]\} .
$$

Com essas relações assim definidas, é possível mostrar a manutenção de todos os resultados usuais da Teoria dos Conjuntos, como:

- $A_{D} \cap B_{D}=B_{D} \cap A_{D} ; A_{D} \cup B_{D}=B_{D} \cup A_{D}$.

- $A_{D} \cup A_{D}=A_{D} \cap A_{D}=A_{D}$.

- $A_{D} \cup\left(B_{D} \cup C_{D}\right)=\left(A_{D} \cup B_{D}\right) \cup C_{D} ; A_{D} \cap\left(B_{D} \cap\right.$ $\left.C_{D}\right)=\left(A_{D} \cap B_{D}\right) \cap C_{D}$.

- $A_{D} \cup \emptyset=A_{D} ; A_{D} \cap \emptyset=\emptyset$.

- $A_{D} \cap U=A_{D} ; A_{D} \cup U=U$.

- $\neg \neg\left(A_{D}\right)=A_{D}$.

- $\neg\left(A_{D} \cup B_{D}\right)=\left(\neg A_{D}\right) \cap\left(\neg B_{D}\right) ; \neg\left(A_{D} \cap B_{D}\right)=$ $\left(\neg A_{D}\right) \cup\left(\neg B_{D}\right)$.

- $\neg(\emptyset)=U ; \neg(U)=\emptyset$.

- Mas, note que $A \cap \bar{A} \neq \emptyset$ e $A \cup \bar{A} \neq U$, quando as funções max-min são utilizadas.

As variáveis linguísticas possuem valores que são descritos por conjuntos fuzzy, representados por funções de pertinência, conforme mostrado na Figura 3 .

Pode-se, ainda, lançar mão dos transformadores difusos 36 que funcionam como adjetivos ou advérbios em linguagem natural. Sua função é dar graus difusos para os conceitos (também difusos) que se deseje usar. Notese que

O processo de "escultura" da curva de pertinência é antes uma arte do que uma ciência, pois a adequação da forma final é avaliada de forma predominantemente subjetiva, dando ampla margem de criatividade ao projetista, tendo em vista o grande espectro de transformadores que poderão alcançar o fim desejado [36, p. 50].

\footnotetext{
1 O uso das funções mínimo e máximo é uma das possibilidades. Há inúmeras outras que podem, a depender do problema, levar a melhores modelagens. Isso deve ser objeto de atenção de quem projeta o modelo. Na modelagem em questão, quando necessário, serão utilizadas apenas funções max-min.
} 


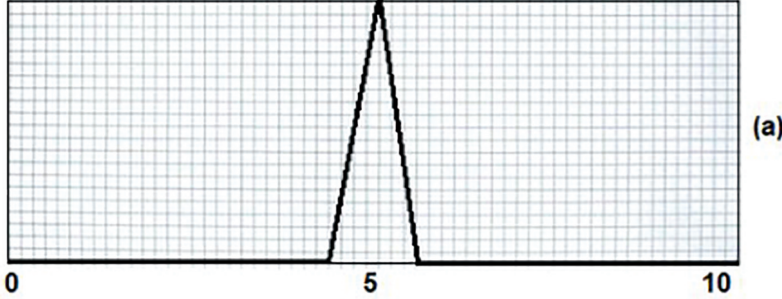

(a)
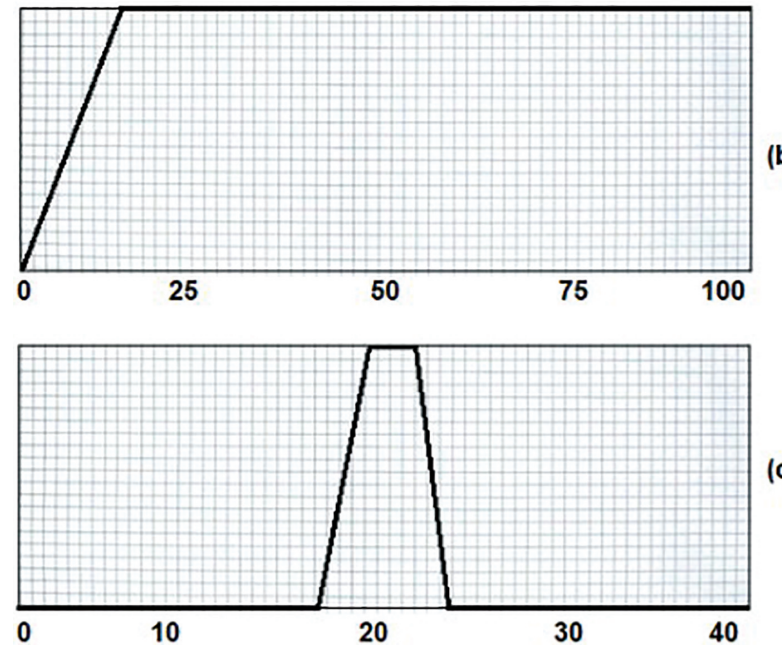

(c)

Figura 3: Algumas funções de pertinência relativas aos conceitos difusos (a) "aproximadamente cinco"; (b) "atrasado" e (c) "temperatura amena".

Fonte: elaboração própria (2021).

Um exemplo de transformador difuso é aquele que representa o advérbio "muito", não raro implementado pela função $f(x)=x^{n}$, com $n>1$.

As funções de pertinência podem ter diferentes formas e, de fato, é como se introduz a semântica das variáveis linguísticas, isto é, seu significado. Na Figura 3(b), por exemplo, a declividade da reta seria, possivelmente, diferente para um brasileiro e um britânico.

Como se deseja falar coisas no universo do discurso implementado pelas variáveis linguísticas, deve-se lançar mão de proposições fuzzy. Tais proposições podem ser da forma predicativa $V L$ é $A$, em que $V L$ é uma variável linguística e $A$ é um conjunto fuzzy. Proposições fuzzy do tipo anterior podem ser combinadas em proposições fuzzy mais complexas com o uso dos conectivos $\wedge, \vee$ e ᄀ ("e", "ou" e "não"), além da implicação fuzzy $\rightarrow$. Essas proposições complexas são expressas por relações fuzzy (e.g., $R_{A \wedge B}$ ) em que $\wedge, \vee$ e $\neg$ são mapeados nas operações já apresentadas acerca de interseção, união e complementar dos conjuntos fuzzy. O conectivo relativo à implicação é o responsável por inserir as regras linguísticas, e é representado por $R_{A \rightarrow B^{\prime}}$, com a função de pertinência $\chi[A \rightarrow B]=f_{\rightarrow}(\chi[A], \chi[B])$, em que $f \rightarrow$ é o operador de implicação. Assim, podemos definir (a partir do resultado da lógica formal $(p \rightarrow q) \Longleftrightarrow$ $[(\neg p) \vee q])$ a função característica para a implicação como

$$
\chi[A \rightarrow B](x, y)=\max \{1-\chi[A](x) ; \chi[B](y)\} .
$$

Com a implicação fuzzy, pode-se definir uma inferência fuzzy, como sendo dada pela sequência (modus ponens):

$$
\left(x \text { é } A^{\prime}\right) \wedge(x \text { é } A \rightarrow y \text { é } B) \rightarrow\left(y \text { é } B^{\prime}\right),
$$

em que o $A^{\prime}$ da premissa não é o $A$ do antecedente da primeira implicação, assim como o consequente da primeira implicação, $B$, não é o mesmo que a consequência final $B^{\prime}$ 37.

Assim, a função de pertinência do consequente, $\chi\left[B^{\prime}\right](y)$ é obtida a partir de uma composição fuzzy que generaliza a sequência anterior, isto é,

$$
\chi\left[B^{\prime}\right](y)=\max \{\chi[A] \wedge \chi[A \rightarrow B](x, y)\}
$$

em que o operador é dado da maneira já indicada. Vale notar que, quando o antecedente $\left(x\right.$ é $A^{\prime}$ ) é dado por um valor sharp (como será o caso - entradas sharp do tipo $\chi\left[A^{\prime}\right](x)=1$ se $x=x^{\prime}$ e zero caso contrário), a implicação difusa se simplifica para

$$
\chi\left[B^{\prime}\right]=\chi[A \rightarrow B]\left(x^{\prime}, y\right)=\max \left\{1-\chi A\left(x^{\prime}\right), \chi[B](y)\right\}
$$

Essa definição, a despeito de ser absolutamente correta, pode ensejar problemas de interpretação em situações de controle, razão pela qual se prefere usar a relação

$$
\chi\left[B^{\prime}\right]=\chi[A \rightarrow B](x, y)=\min \left\{\chi[A]\left(x^{\prime}\right), \chi[B](y)\right\}
$$

que representa, simplesmente, a mesma regra que a definida para a conjunção.

Do ponto de vista operativo ou computacional, a inferência fuzzy (ou seja, a regra) será disparada se houver grau de similaridade entre a premissa $A^{\prime}$ e o antecedente da regra, dando como resultado um consequente com grau de similaridade não nulo, em relação ao consequente da regra. Assim, o grau de pertinência de $x^{\prime}$ em $A$ estabelece o grau de ativação de determinada regra: quanto mais a entrada for compatível com o antecedente da regra, mais peso terá o seu consequente no resultado final [37]. De fato, se $\chi[A](x)=1$, então $B^{\prime}$ será o próprio conjunto $B$.

Esquematicamente, um sistema de inferência fuzzy tem as características apresentadas na Figura 4

O último passo, portanto, de acordo com a Figura 4 é o processo de defuzzificação, para casos em que é necessário apresentar um resultado sharp. O conjunto fuzzy de saída, neste trabalho, é dado pelas duas variáveis linguísticas Compreensão Nuclear do subsunçor (CN) e Compreensão Periférica do subsunçor (CP). Cada uma dessas variáveis irá ser defuzzificada usandose a estratégia do centro de gravidade, segundo a qual calcula-se o centro de gravidade associado à função de pertinência da variável fuzzy desejada e atribui-se a ela este valor único.

A utilização da lógica fuzzy em contexto educacional, no Brasil, é particularmente desenvolvida em trabalhos que relacionam aprendizagem, avaliação e permanência 38 41. O elemento identitário dessas produções 


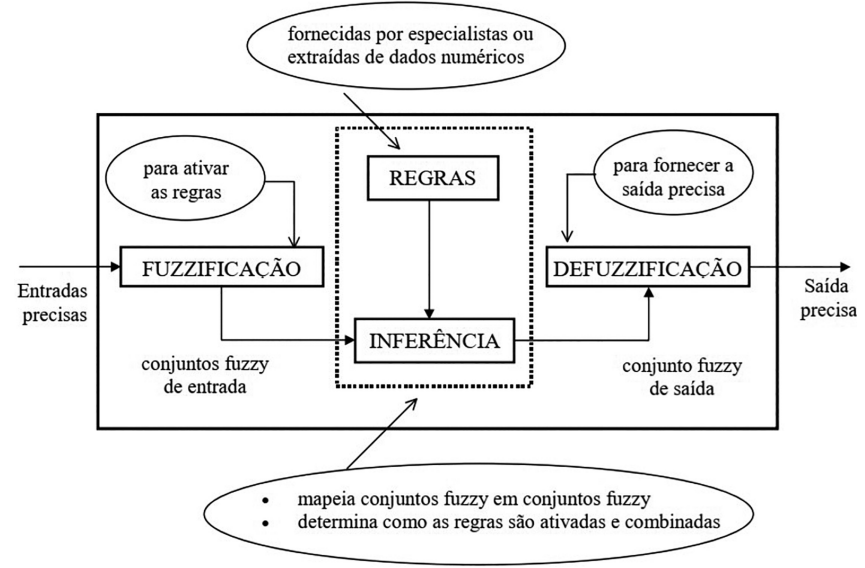

Figura 4: Esquema geral de um sistema de inferência fuzzy. Fonte: [37, p. 26].

é aquele que busca associar tais resultados a coleções de parâmetros ou variáveis que permitem transições graduais entre as relações de existência ou de correlação, em detrimento à assunção de um mero caráter dual de fatores intervenientes em determinado conjunto (como a de pertencimento ou não pertencimento). Assim, na perspectiva desses trabalhos, a aprendizagem de conceitos pode ser um objeto que envolve variáveis quantitativas e qualitativas, associado a funções de pertinência e a lógicas inferenciais, tal qual pretendemos explicitar no modelo teórico para levantamento e organização de subsunçores na aprendizagem de física.

\section{Modelo teórico para levantamento e organização de subsunçores}

Ainda que a implementação computacional realizada inclua diversas flexibilizações, como modelagem com diferentes funções de pertinência, ou mesmo diferentes parametrizações destas, apresentamos, a seguir, como forma de exemplificação, uma simplificação do modelo adotado neste trabalho:

\section{Variáveis Linguísticas:}

(a) Marcação do estudante (variável sharp ME): tal que (marcou a alternativa $\rightarrow$ 1; não marcou a alternativa $\rightarrow 0$ ).

(b) Relevância do item (variável fuzzy $\mathrm{RI})$ : (B = baixa; $\mathrm{M}=$ média; $\mathrm{A}=$ alta).

(c) Importância da alternativa (variável fuzzy IA $):(\mathrm{AN}=$ alta negativa; $\mathrm{BN}=$ baixa negativa; $\mathrm{BP}=$ baixa positiva; $\mathrm{AP}=$ alta positiva): tais graus representam que a alternativa afirma:

i. AN: erro grosseiro quanto ao que é o subsunçor.

ii. BN: erro sutil quanto ao que é o subsunçor. iii. BP: adequação sutil quanto ao que é o subsunçor.

iv. AP: adequação forte quanto ao que é o subsunçor.

(d) Compreensão nuclear do subsunçor (variável fuzzy $\mathrm{CN})$ : $(\mathrm{B}=$ baixa; $\mathrm{M}=$ média; $\mathrm{A}=$ alta $)$.

(e) Compreensão periférica do subsunçor (variável fuzzy $\mathrm{CP})$ : ( $\mathrm{B}=$ baixa, $\mathrm{M}=$ média, $\mathrm{A}=$ alta).

2. Funções de pertinência para cada variável, que serão:

(a) RI, CN, CP:

$$
\begin{gathered}
\chi[A](x)=\left(1+9(x-1)^{2}\right)^{-1} \\
\chi[M](x)=\left(1+30(x-0,5)^{2}\right)^{-1} \\
\chi[B](x)=\left(1+9 x^{2}\right)^{-1}
\end{gathered}
$$

(b) IA:

$$
\begin{gathered}
\chi[A P](x)=\left(1+9(x-1)^{2}\right)^{-1} \\
\chi[B P](x)=\left(1+30(x-0,5)^{2}\right)^{-1} \\
\chi[B N](x)=\left(1+30(x+0,5)^{2}\right)^{-1} \\
\chi[A N](x)=\left(1+9(x+1)^{2}\right)^{-1}
\end{gathered}
$$

As funções de pertinência estão apresentadas na Figuras 5 e 6

\section{Regras:}

As regras a seguir devem ser impostas a cada alternativa do item, para se chegar aos valores das variáveis linguísticas $\mathrm{CN}$ e $\mathrm{CP}$, representando a compreensão nuclear do subsunçor e sua compreensão periférica. Um critério adicional de ativação das regras é que o antecedente 'IA é' dê como resultado um número maior ou igual a 0,15 (uma vez que as funções utilizadas são contínuas). Assim, tem-se:

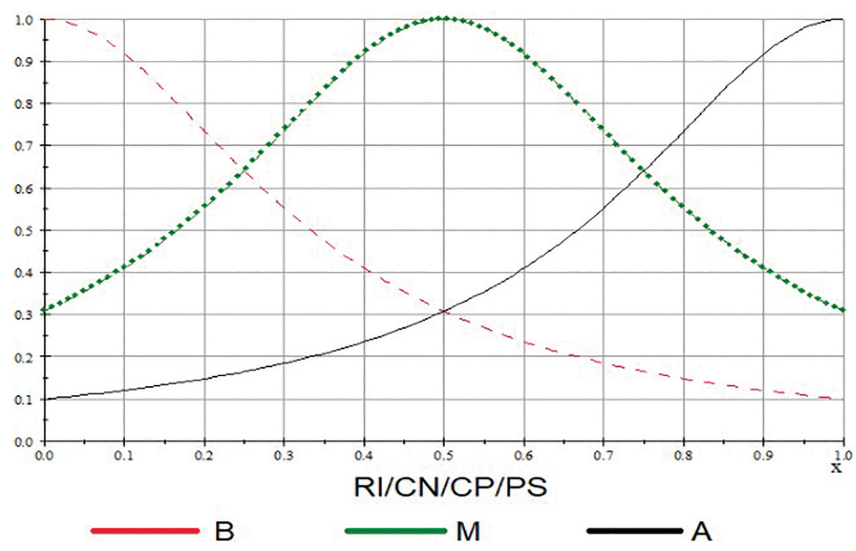

Figura 5: Funções de pertinência para $B, M$ e $A$, para as variáveis RI, CN, CP e OS.

Fonte: elaboração própria (2021). 


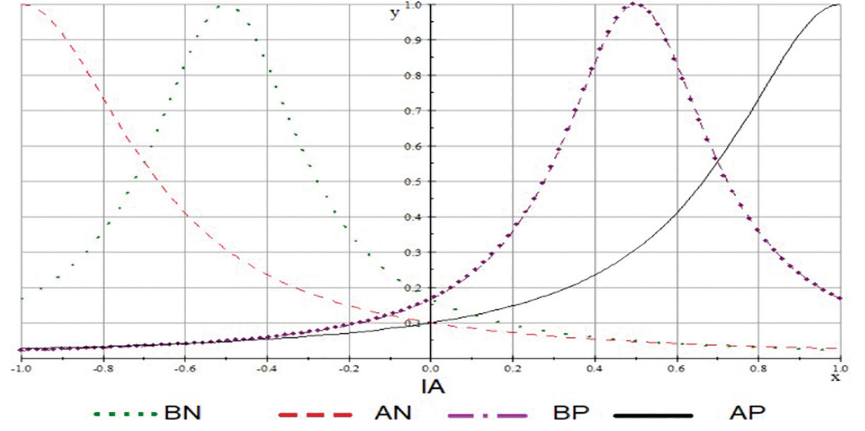

Figura 6: Funções de pertinência para BN, NA, BP e AP da variável IA.

Fonte: elaboração própria (2021).

(a) Para CN:

i. $\mathrm{Se} \mathrm{ME}=1$ \& IA é AP, então CN é MUITO A.

ii. Se $\mathrm{ME}=0$ \& IA é $\mathrm{AP}$, então $\mathrm{CN}$ é MUITO $\mathrm{B}$.

iii. $\mathrm{Se} \mathrm{ME}=1$ \& IA é BP, então CN é M. iv. $\mathrm{Se} \mathrm{ME}=0$ \& IA é BP, então CN é B.

v. Se $\mathrm{ME}=1 \&$ IA é $\mathrm{AN}$, então $\mathrm{CN}$ é B. vi. Se $\mathrm{ME}=0$ \& IA é $\mathrm{AN}$, então $\mathrm{CN}$ é $\mathrm{A}$. vii. Se $\mathrm{ME}=1$ \& IA é $\mathrm{BN}$, então $\mathrm{CN}$ é POUCO B.

viii. Se $\mathrm{ME}=0$ \& IA é $\mathrm{BN}$, então $\mathrm{CN}$ é $\mathrm{M}$.

(b) Para CP:

i. Se ME $=0$ \& IA é AN, então CP é MUITO A.

ii. Se $\mathrm{ME}=1 \&$ IA é AN, então CP é MUITO B.

iii. Se $\mathrm{ME}=0$ \& IA é BN, então CP é M.

iv. $\mathrm{Se} \mathrm{ME}=1$ \& IA é BN, então CP é B.

v. Se $\mathrm{ME}=0$ \& IA é AP, então CP é B.

vi. $\mathrm{Se} \mathrm{ME}=1 \& \mathrm{IA}$ é $\mathrm{AP}$, então $\mathrm{CP}$ é $\mathrm{A}$.

vii. $\mathrm{Se} \mathrm{ME}=0$ \& IA é BP, então CP é POUCO B.

viii. Se $\mathrm{ME}=1$ \& IA é $\mathrm{BP}$, então $\mathrm{CP}$ é $\mathrm{M}$.

A aplicação das regras anteriores irá, para cada alternativa, gerar um conjunto difuso para $\mathrm{CN}$ e CP. Entretanto, deseja-se saber a presença ou ausência do subsunçor, que está vinculada ao item. Para tanto, fazse um $\vee$ fuzzy entre todas as regras CN e outro $\vee$ fuzzy entre todas as regras $\mathrm{CP}$.

Finalmente, realiza-se a defuzzificação de $\mathrm{CN}$ e $\mathrm{CP}$, para compreender o grau de presença (nessas categorias) do subsunçor na estrutura cognitiva do estudante.

\subsection{Exemplo}

Supõe-se, a título de exemplo, que determinado item possua cinco alternativas com os seguintes valores de importância, atribuídos pelo professor:

$$
\text { a) } 0,4 ; b) 0,8 ; c)-0,6 ; d)-0,9 ; e) 0,3
$$

e que as marcações $(\mathrm{ME}=1)$ do estudante foram $(\mathrm{b}),(\mathrm{c})$ e (e), deixando em branco $(\mathrm{ME}=0)$ (a) e (d). Assim, para as alternativas, são ativadas as regras referentes a:

(a) $(\mathrm{ME}=0)$ e IA é $\mathrm{AP}(0,24)$ e IA é $\mathrm{BP}(0,77)$ [regras: a.ii, b.v, a.iv e b.vii].

(b) $(\mathrm{ME}=1)$ e IA é $\mathrm{AP}(0,74)$ e IA é $\mathrm{BP}(0,27)$ [regras: a.i, b.vi, a.iii e b.viii].

(c) $(\mathrm{ME}=1)$ e IA é $\mathrm{AN}(0,41)$ e IA é $\mathrm{BN}(0,77)$ [regras: a.v, b.ii, a.vii e b.iv].

(d) $(\mathrm{ME}=0)$ e IA é $\mathrm{AN}(0,92)$ e IA é $\mathrm{BN}(0,17)$ [regras: a.vi, b.i, a.viii e b.iii].

(e) $(\mathrm{ME}=1)$ e IA é $\mathrm{AP}(0,18)$ e IA é $\mathrm{BP}(0,45)$ [regras: a.i, b.vi, a.iii e b.viii].

- Para a alternativa (a), $x=0,4$, e tem-se que $\chi[A P](0,4)=0,24$ e $\chi[B P](0,4)=0,77$. Aplicase, para AP, a regra a.ii que diz que $C N$ é MUITO baixo. Então, tem-se que aplicar o transformador MUITO (aqui, utiliza-se a função $f(x)=x^{3}$ ) a $\chi[B](x)$. Fica-se com a função $f(\chi[B](x))=(1+$ $\left.9 x^{2}\right)^{-3}$. A inferência, como o valor de $x$ é sharp, resulta em

$$
\begin{aligned}
\chi[C N](y) & =\min (\chi[A P](x) ; f(\chi[B](y))) \\
& =\min \left(0,24 ;\left(1+9 y^{2}\right)^{-3}\right)
\end{aligned}
$$

Para BP, a regra a.iv implica que

$$
\chi[C N](y)=\max \left(0,77 ;\left(1+9 x^{2}\right)^{-1}\right) .
$$

Pode-se resumir o processo nas seguintes relações:

\begin{tabular}{|l|l|}
\hline AP regra a.ii & $\min \left(0,24 ;\left(1+9 y^{2}\right)^{-3}\right)$ \\
\hline BP regra a.iv & $\min \left(0,77 ;\left(1+9 y^{2}\right)^{-1}\right)$ \\
\hline
\end{tabular}

- Para a alternativa (b), $x=0,8, \chi[A P](0,8)=0,74$ e $\chi[B P](0,8)=0,27$. Tem-se:

\begin{tabular}{|l|l|}
\hline AP regra a.i & $\min \left(0,74 ;\left(1+9 y^{2}\right)^{-3}\right)$ \\
\hline BP regra a.iii & $\min \left(0,27 ;\left(1+30 y^{2}\right)^{-1}\right)$ \\
\hline
\end{tabular}

- Para a alternativa (c), $x=-0,6, \chi[A N](-0,6)=$ 0,41 e $\chi[B P](-0,6)=0,77$, tem-se (função POUCO expressa por $\left.f(x)=x^{-1 / 3}\right)$ :

\begin{tabular}{|l|l|}
\hline AN regra a.v & $\min \left(0,41 ;\left(1+9 y^{2}\right)^{-1}\right)$ \\
\hline BP regra a.vii & $\min \left(0,77 ;\left(1+9 y^{2}\right)^{-1 / 3}\right)$ \\
\hline
\end{tabular}


Para a alternativa (d), $x=-0,9, \chi[A N](-0,9)=$ 0,92 e $\chi[B N](-0,9)=0,17$, tem-se:

\begin{tabular}{|l|l|}
\hline AN regra a.vi & $\min \left(0,92 ;\left(1+9(y-1)^{2}\right)^{-1}\right)$ \\
\hline BN regra a.viii & $\min \left(0,17 ;\left(1+30(y-0,5)^{2}\right)^{-1}\right)$ \\
\hline
\end{tabular}

- Para a alternativa (e), $x=0,3, \chi[A P](0,3)=0,18$ e $\chi[B P](0,3)=0,45$, tem-se:

\begin{tabular}{|l|l|}
\hline AP regra a.i & $\min \left(0,18 ;\left(1+9(y-1)^{2}\right)^{-3}\right)$ \\
\hline BP regra a.iii & $\min \left(0,45 ;\left(1+30(y-0,5)^{2}\right)^{-1}\right)$ \\
\hline
\end{tabular}

- Finalmente, para obter a função de pertinência $\chi[C N](y)$ que representa o item, faz-se a união fuzzy dos cinco resultados, resultando em

$$
\begin{gathered}
\chi[C N](y)=\max \left(\min \left(0,24 ;\left(1+9 y^{2}\right)^{-3}\right) ;\right. \\
\min \left(0,77 ;\left(1+9 y^{2}\right)^{-1}\right) ; \\
\min \left(0,74 ;\left(1+9(y-1)^{2}\right)^{-3}\right) ; \\
\min \left(0,27 ;\left(1+30(y-0,5)^{2}\right)^{-1}\right) ; \\
\min \left(0,41 ;\left(1+9 y^{2}\right)^{-1}\right) ; \\
\min \left(0,77 ;\left(1+9 y^{2}\right)^{-1 / 3}\right) ; \\
\min \left(0,92 ;\left(1+9(y-1)^{2}\right)^{-1}\right) \\
\min \left(0,17 ;\left(1+30(y-0,5)^{2}\right)^{-1}\right) \\
\min \left(0,18 ;\left(1+9(y-1)^{2}\right)^{-3}\right) \\
\left.\min \left(0,45 ;\left(1+30(y-0,5)^{2}\right)^{-1}\right)\right)
\end{gathered}
$$

representado na Figura 7 (linha tracejada), em que já foram apresentados os cálculos feitos para CP (linha sólida), utilizando-se das mesmas ideias para obter a função de pertinência:

$$
\begin{gathered}
\chi[C N](y)=\max \left(\min \left(0,24 ;\left(1+9 y^{2}\right)^{-1}\right) ;\right. \\
\min \left(0,77 ;\left(1+9 y^{2}\right)^{-1 / 3}\right) ; \\
\min \left(0,74 ;\left(1+9(y-1)^{2}\right)^{-1}\right) ; \\
\min \left(0,27 ;\left(1+30(y-0,5)^{2}\right)^{-1}\right) \\
\min \left(0,41 ;\left(1+9 y^{2}\right)^{-1 / 3}\right) \\
\min \left(0,77 ;\left(1+9 y^{2}\right)^{-1}\right) \\
\min \left(0,92 ;\left(1+9(y-1)^{2}\right)^{-3}\right) \\
\min \left(0,17 ;\left(1+30(y-0,5)^{2}\right)^{-1}\right) \\
\min \left(0,18 ;\left(1+9(y-1)^{2}\right)^{-1}\right) \\
\left.\min \left(0,45 ;\left(1+30(y-0,5)^{2}\right)^{-1}\right)\right)
\end{gathered}
$$

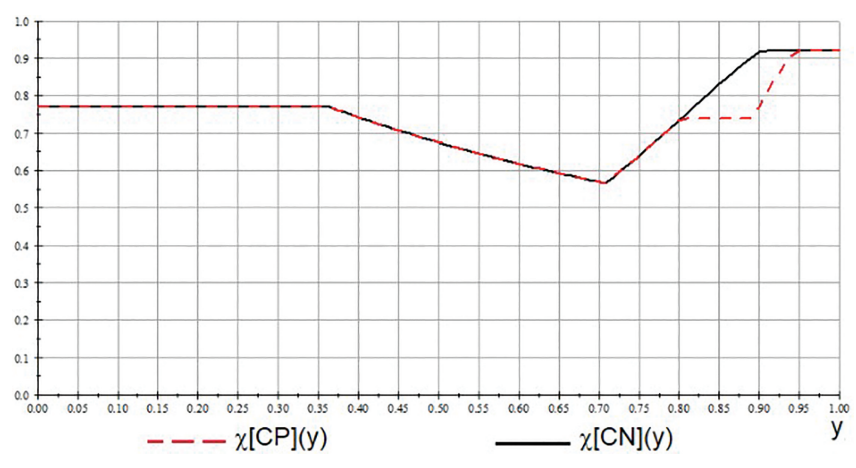

Figura 7: Funções de pertinência do item exemplo para as variáveis fuzzy Conhecimento Periférico $(\mathrm{CP})$ e Conhecimento Nuclear (CN).

- Como defusificação, calcula-se o centro de gravidade das figuras como as integrais

$$
\begin{aligned}
& C N=\frac{\int y \chi[C N](y) d y}{\int \chi[C N](y) d y}=\frac{0,374}{0,741}=0,504 \\
& C P=\frac{\int y \chi[C P](y) d y}{\int \chi[C P](y) d y}=\frac{0,363}{0,729}=0,498 .
\end{aligned}
$$

\section{Proposta de aplicativo para implementação técnica do modelo teórico para levantamento e organização de subsunçores}

A partir dos desenvolvimentos teóricos anteriores, foi

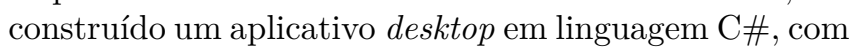
o propósito de implementar tecnicamente a inferência difusa, construída a partir dos inputs do professor (graus de pertinência dos itens e de importância das alternativas) e dos estudantes (suas marcações), tendo em vista o levantamento e a organização de subsunçores ${ }^{2}$

$\mathrm{O}$ aplicativo calcula os valores finais das variáveis semânticas CN e CP para cada item, para cada estudante. Para tanto, os dados devem estar dispostos em uma planilha Excel da maneira indicada na Figura 8

1. Primeira linha:

A) Primeira coluna: inserir o título do item (e.g., 'ITEM 1').

B) Segunda coluna: número de alternativas do item (e.g., 5, 8, ...). Pode haver qualquer número de alternativas; quanto maior, maior a probabilidade de se discriminar entre os estudantes.

C) Terceira coluna: inserir termo "menor". Nela, o sistema calculará o menor valor possível de

\footnotetext{
$\overline{2} \mathrm{O}$ instalador do programa pode ser baixado no endereço encurtador.com.br/afwz1. O programa é oferecido gratuitamente na forma as is e não implica responsabilidade dos autores relativamente a efeitos que possa causar nas máquinas em que for instalado.
} 


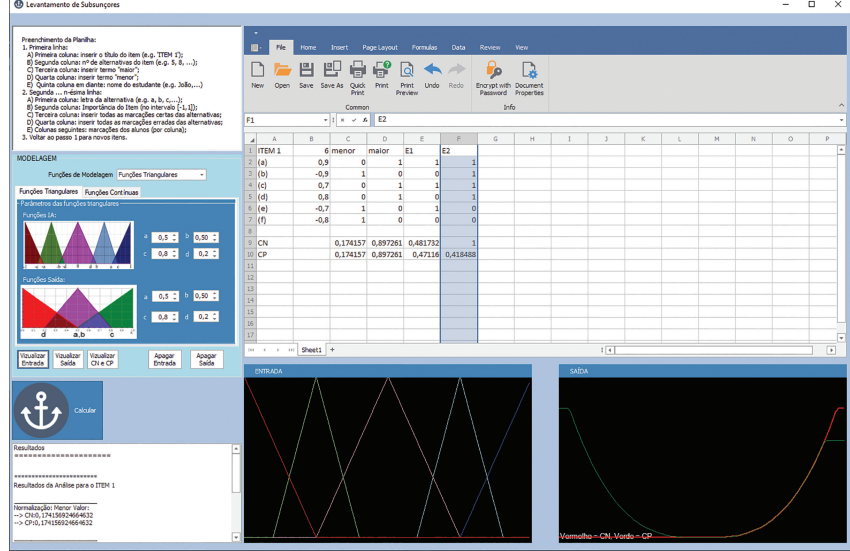

Figura 8: Sistema computacional para o cálculo dos valores das variáveis semânticas $\mathrm{CN}$ e CP.

Fonte: elaboração própria (2021).

CN e CP para o item em questão. Este cálculo é importante, pois itens diferentes possuem valores máximos de $\mathrm{CN}$ e $\mathrm{CP}$ diferentes e, para se poder compará-los, eles devem ser normalizados.

D) Quarta coluna: inserir termo "maior". Nela, o sistema calculará o maior valor possível de $\mathrm{CN}$ e $\mathrm{CP}$ para o item, pelas razões já apresentadas.

E) Quinta coluna em diante: nome do estudante (e.g., João, ...): deve ser inserido um identificador qualquer, seja o nome, seja a matrícula.

2. Segunda ... n-ésima linha: entrada das alternativas do item.

A) Primeira coluna: letra da alternativa (e.g. a, $b, c, \ldots)$.

B) Segunda coluna: Importância do Item (IA) (no intervalo $[-1,1])$ : é o input do professor.

C) Terceira coluna: inserir todas as marcações erradas das alternativas para o sistema calcular o valor mínimo de CN e CP para o item em questão.

D) Quarta coluna: inserir todas as marcações corretas das alternativas para o sistema calcular o valor máximo de CN e CP para o item em questão.

E) Colunas seguintes: marcações dos estudantes (por coluna). Deve-se preencher a célula com 1 , se o estudante marcou a alternativa, ou 0 , se ele não a marcou.

F) Cada item deve ser colocado em um Worksheet diferente. No exemplo da Figura 8 , são ilustrados dois itens. Um no "Sheet1" e o outro no "Sheet2";
3. Voltar ao passo 1 para incluir, em novos Worksheets, novos itens.

Todo o processo de construção da planilha pode ser feito fora do sistema, para posterior processamento, ou diretamente nele. O sistema funciona sem necessidade de conexão com a internet.

Uma vez lida a planilha e apresentada para o professor, basta clicar o botão "calcular", para que cada valor de CN e CP seja apresentado nas duas linhas subsequentes aos dados, por estudante, como ilustra a Figura 8 .

O programa não faz, ainda, automaticamente a etapa de organização avançada de subsunçores, cabendo ao professor, de posse dos valores de CN e CP, dar a ela andamento. Entretanto, tal etapa já está sendo considerada para futura implementação.

\subsection{Ilustração do Modelo}

Considere uma situação hipotética, em que o professor busca ensinar estática aos seus estudantes. Para tanto, constrói, primeiramente, um mapa conceitual do campo, no qual aparecem os conceitos de torque, força, aceleração, velocidade, massa, deslocamento, distância, tempo, dentre outros característicos da área de conhecimento. A partir desse mapa, que o leitor pode inferir por si próprio, o professor deseja saber a intensidade com que o subsunçor força está presente na estrutura cognitiva de cada estudante. Para tanto, ele elabora o item mostrado no Quadro 1, ao qual ele atribui relevância igual a 0.9 (alta, portanto) acerca do subsunçor "força" e suas relações com outros subsunçores importantes:

Na Tabela 11 muitas outras alternativas poderiam ser incluídas, formando uma investigação completa dos subsunçores dos estudantes no que concerne ao conceito de força e sua relação com contextos necessários ao seu uso na definição de "equilíbrio", conceito central da Estática newtoniana.

Suponhamos, agora, que dois estudantes, E1 e E2, apresentaram as seguintes marcações: E1: marcou (a), (c) e (e); E2: marcou (a), (b), (c) e (d). Ao rodarmos o sistema (com regras distintas das que foram apresentadas), chegamos aos escores apresentados na Figura 9

As duas primeiras colunas servem para normalizar os resultados, que passam a contar no intervalo $[0,1]$ (note que, pela coluna "C", uma marcação totalmente errada implicaria escore 0.174 , aproximadamente, enquanto que uma marcação totalmente correta implicaria escore 0,897, aproximadamente. Como o estudante E2 marcou todas as alternativas corretas, seu escore $\mathrm{CN}$ foi igual a 1, indicando presença dos subsunçores nucleares. Entretanto, sua marcação equivocada da alternativa (b) implicou em uma redução considerável, dada a importância da alternativa, do escore CP final, indicando a necessidade de desenvolvimento e organização do subsunçor na sua articulação periférica. O estudante E2, 
Tabela 1: Item hipotético para levantamento do subsunçor "força", na sua relação com o subsunçor "torque" e com o subsunçor "movimento".

\begin{tabular}{|c|c|c|c|}
\hline Texto do Item & \multicolumn{3}{|c|}{$\begin{array}{l}\text { O conceito de força cumpre, na Mecânica newtoniana, papel central em todas } \\
\text { as suas áreas de aplicação e, em particular, no campo da Estática. }\end{array}$} \\
\hline Comando do item & \multicolumn{3}{|c|}{$\begin{array}{l}\text { Acerca do conceito de "força" na Mecânica newtoniana, julgue os itens a seguir } \\
\text { (marcando os que julgar corretos, e deixando em branco os que julgar incorretos). }\end{array}$} \\
\hline Importância* & Indicador do item & Texto da Alternativa & Justificativa* \\
\hline$(0.9)$ & (a) & $\begin{array}{l}\text { O conceito de força é um } \\
\text { conceito intimamente ligado } \\
\text { ao conceito de aceleração. }\end{array}$ & $\begin{array}{l}\text { Busca estabelecer se o } \\
\text { estudante tem noção da Segunda } \\
\text { Lei de Newton, necessária para } \\
\text { a construção formal do conceito } \\
\text { de equilíbrio. }\end{array}$ \\
\hline$(-0.9)$ & (b) & $\begin{array}{l}\mathrm{Na} \text { ausência de força externa } \\
\text { resultante, um corpo estará } \\
\text { em repouso. }\end{array}$ & $\begin{array}{l}\text { Busca estabelecer se o estudante } \\
\text { entende que repouso e MRU são } \\
\text { equivalentes na Mecânica } \\
\text { newtoniana. }\end{array}$ \\
\hline$(0.7)$ & (c) & $\begin{array}{l}\text { Forças internas a um sistema } \\
\text { não alteram o estado de } \\
\text { movimento do sistema. }\end{array}$ & $\begin{array}{l}\text { Busca estabelecer se o estudante } \\
\text { entende a relação entre forças } \\
\text { internas e externas. }\end{array}$ \\
\hline$(0.8)$ & (d) & $\begin{array}{l}\text { O módulo do torque é } \\
\text { representado pelo produto } \\
\text { da componente } \\
\text { perpendicular da força } \\
\text { pela distância de seu } \\
\text { ponto de aplicação ao } \\
\text { ponto em que este torque } \\
\text { é considerado. }\end{array}$ & $\begin{array}{l}\text { Busca estabelecer se o estudante } \\
\text { é capaz de calcular um torque em } \\
\text { situações mecânicas usuais. }\end{array}$ \\
\hline$(-0.7)$ & (e) & $\begin{array}{l}\text { Enquanto o torque se } \\
\text { relaciona com o movimento } \\
\text { circular do sistema, em } \\
\text { torno de um ponto, a força } \\
\text { se relaciona com seu } \\
\text { movimento translacional. }\end{array}$ & $\begin{array}{l}\text { Busca estabelecer se o estudante } \\
\text { é capaz de diferenciar os } \\
\text { contextos de uso da noção de } \\
\text { "força" e de "torque". }\end{array}$ \\
\hline$(-0.8)$ & (f) & $\begin{array}{l}\text { O sinal do torque representa } \\
\text { a direção e o sentido em que } \\
\text { um corpo se move, ao } \\
\text { apontar nessa direção e } \\
\text { sentido. }\end{array}$ & $\begin{array}{l}\text { Busca saber se o estudante é } \\
\text { capaz de compreender o } \\
\text { significado de "sinal" na } \\
\text { noção de "torque". }\end{array}$ \\
\hline Total $=0.0$ & & & \\
\hline
\end{tabular}

*Não aparecem para os estudantes. Fonte: elaboração própria (2021).

por sua vez, apresentou marcações (e não marcações) equivocadas, resultando em escores $\mathrm{CN}$ e $\mathrm{CP}$ baixos. Note que cabe ao professor inserir um nível mínimo de escore que indique a necessidade de fortalecimento do subsunçor na estrutura cognitiva de cada estudante. Eventualmente, o nível 0,45 poderia ter sido selecionado, indicando que o estudante E2, apesar de seus equívocos, não precisaria ser submetido ao fortalecimento de seus subsunçores (nucleares ou periféricos), mas o estudante E1 sim, com relação aos subsunçores periféricos.

Apesar dessa busca pela materialização dos subsunçores, e pelo esforço aqui presente em ilustrar tamanha abstração, há ocasiões em que eles não são acessíveis em primeira instância, por mais bem desenhados que sejam a estruturação da matéria de conhecimento e o processo instrucional. É precisamente nessa contingência que Ausubel 2] discute a possibilidade de construi-los ou modulá-los por meio de um organizador avançado, caracterizado como um material introdutório, estável, relevante e claro.

Esse recurso é, relativamente a um subsunçor, mais abstrato, genérico e inclusivo do conhecimento cujo acesso se pretende; ademais, é mais efetivo na preparação da estrutura cognitiva. Os princípios de sua formulação e uso envolvem três fatores principais: a) a identificação da importância potencial de determinadas ideias para a cognição; b) a clarividência de materiais gerais e inclusivos implicam possibilidades mais concretas de 


\begin{tabular}{|r|l|r|r|r|r|r|r|}
\hline & \multicolumn{1}{|c|}{ A } & B & \multicolumn{1}{c|}{ C } & \multicolumn{1}{c|}{ D } & E & \multicolumn{2}{|c|}{$F$} \\
\hline 1 & ITEM 1 & 6 & menor & maior & E1 & E2 \\
\hline 2 & (a) & 0,9 & 0 & 1 & 1 & 1 \\
\hline 3 & (b) & $-0,9$ & 1 & 0 & 0 & 1 \\
\hline 4 & (c) & 0,7 & 0 & 1 & 1 & 1 \\
\hline 5 & (d) & 0,8 & 0 & 1 & 0 & 1 \\
\hline 6 & (e) & $-0,7$ & 1 & 0 & 1 & 0 \\
\hline 7 & (f) & $-0,8$ & 1 & 0 & 0 & 0 \\
\hline 8 & & & & & & & \\
\hline 9 & CN & & 0,174157 & 0,897261 & 0,481732 & 1 \\
\hline 10 & CP & & 0,174157 & 0,897261 & 0,47116 & 0,418488 \\
\hline
\end{tabular}

Figura 9: Entradas e Saídas do sistema de cálculo dos subsunçores dos estudantes E1 e E2.

Fonte: elaboração própria (2021).

elucidação e de integração cognitiva; e, por fim, c) que eles são tanto mais qualificados quanto possível clarificar as suas relações com a estrutura do conhecimento e as formas de aprendizagem [29].

A identificação de demandas cognitivas por organização avançada, bem como o desenvolvimento de materiais potencialmente significativos, é etapa sequencial e intrínseca ao levantamento e à organização de subsunçores. Entretanto, trata-se de um processo de natureza peculiar e que incorpora princípios e leis de formação e funcionamento próprios, cuja formulação escapa ao escopo e às limitações desta discussão, ainda que à vista para as que buscarão desdobrá-la.

\section{Considerações Finais}

No modelo teórico e na técnica que dele decorre, como apresentado e exemplificado, há, como é fácil notar, diversos elementos que dependem, em maior ou menor grau, da subjetividade do professor. Assim, sabese que dois professores desenvolverão, para o mesmo campo conceitual e as mesmas atividades, mapas mentais relativamente distintos; também é esperado que as atribuições de relevância divirjam em algum grau, assim como diferirão as atribuições de adequação para as alternativas, nos itens fechados de escolha múltipla. Esses são os elementos irredutíveis de subjetividade que a técnica aqui apresentada não apenas não considera deletérios, como assume que representam a própria riqueza do processo de ensino e aprendizagem, não sendo seu objetivo, de modo algum, remover tal subjetividade.

O que se acredita e se buscou evidenciar, entretanto, é que devem, igualmente, haver convergências para cada um desses elementos que, uma vez sistematizados em uma técnica, suscitam uma prática na qual as diferenças podem concorrer para o enriquecimento mútuo de distintas soluções apresentadas para o momento de ensino e aprendizagem, além de explicitar, sem suprimir, as diversas escolhas possíveis.

Também parece possível, agora, com a aplicação da mesma técnica, com os mesmos mapas conceituais igualmente parametrizados, comparar a presença ou não de subsunçores em diferentes grupos de estudantes, podendo servir como importante elemento diagnóstico também para as escolas e respectivas redes de ensino.

O próprio sistema de regras aqui apresentado pode ser, eventualmente, aprimorado, incluindo-se mais regras e, com isso, uma possível maior lapidação do levantamento dos subsunçores.

Finalmente, com o processo aqui descrito, abre-se a possibilidade de se construir instrumentos de avaliação que realizem, de maneira automática, os processos de levantamento e organização dos subsunçores, implicando na possibilidade de se construir avaliações consistentes com a noção de aprendizagem significativa no contexto de testagens automatizadas e flexibilizadas, baseadas em tecnologias digitais da informação e comunicação (TDIC).

O mínimo que a técnica aqui apresentada fornece é um processo de sistematização que organiza objetivamente elementos de subjetividade e difusividade que comparecem no processo de levantamento de subsunçores e na sua utilização no processo de organização avançada, etapas absolutamente essenciais do processo de ensino e aprendizagem de caráter significativo, mas que não têm recebido atenção proporcional à sua relevância no contexto da aplicação da TAS.

\section{Referências}

[1] D. Ausubel, The psychology of meaningful verbal learning (Grune \& Stratton, New York, 1963).

[2] D. Ausubel, Educational psychology: a cognitive view (Holt, Rinehart \& Winston, New York, 1968).

[3] D. Ausubel, J.D. Novak e H. Hanesian, Educational psychology: a cognitive view (Holt, Rinehart \& Winston, New York, 1978).

[4] J.D. Novak, Uma teoria de educação (Pioneira, São Paulo, 1977).

[5] J.D. Novak, Aprender a criar e utilizar o conhecimento: mapas conceptuais como ferramenta de facilitação nas escolas e empresas (Paralelo Editora, Lisboa, 2000).

[6] J.D. Novak, Learning, Creating, and Using Knowledge: Concept Mapas as Facilitative Tools in Schools and Corporations (Routledge, New York, 2010).

[7] D.B. Gowin, Educating (Cornell University Press, New York, 1981).

[8] M.A. Moreira e E.A.F. Masini, Aprendizagem significativa: a teoria de David Ausubel (Editora Moraes, São Paulo, 1982).

[9] M.A. Moreira e E.A.F. Masini, Aprendizagem significativa: a teoria de David Ausubel (Centauro Editora, 2006, São Paulo), $2^{\mathrm{a}}$ ed.

[10] M.A. Moreira, Uma abordagem cognitivista ao ensino da Física (Editora da Universidade, Porto Alegre, 1983).

[11] M.A. Moreira e B. Buchweitz, Novas estratégias de ensino e aprendizagem (Plátano Edições Técnicas, Lisboa, 1993).

[12] M.A. Moreira, Aprendizagem significativa (Editora da UnB, Brasília, 1999).

[13] M.A. Moreira, Aprendizaje significativo: teoría y práctica (Visor, Madrid, 2000). 
[14] M.A. Moreira, Aprendizaje significativa crítica (Instituto de Física da UFRGS, Porto Alegre, 2005).

[15] M.A. Moreira, Mapas conceituais e diagramas $V$ (Ed. do Autor, Porto Alegre, 2006).

[16] M.A. Moreira, Aprendizagem Significativa: a Teoria e Textos Complementares (Editora da Física, São Paulo, 2011).

[17] M.A. Moreira, Aprendizagem Significativa em Revista 1, 25 (2011)

[18] M.A. Moreira, C. Caballero e P.M.L. Rodríguez, Aprendizaje significativo: interacción personal, progresividad y lenguaje (Universidad de Burgos, Burgos, 2004).

[19] E.A.F. Masini e M.A. Moreira, Aprendizagem significativa: condições para ocorrência e lacunas que levam a comprometimentos (Vetor Editora, São Paulo, 2008).

[20] J. Valadares e M.A. Moreira, A teoria da aprendizagem significativa: sua fundamentação e implementação (Edições Almedina, Coimbra, 2009).

[21] O.L. Silva Filho e M. Ferreira, Revista do Professor de Física 2, 104 (2018).

22] O.L. Silva Filho, M. Ferreira, A.M.M. Polito e A.L.M.B. Coelho, Pesquisa e Debate em Educação 11, e32564 (2021).

[23] F. Frasson, C.E. Laburú e A.F. Zompero, Revista Contexto \& Educação 34, 303 (2019).

[24] L. Bacich, A. Tanzi Neto e F. Trevisani, Ensino híbrido: personalização e tecnologia na educação (Penso, Porto Alegre, 2015).

[25] P. Lévy, As tecnologias da inteligência (Editora 34, Rio de Janeiro, 1993)

[26] V.M. Kenski, em: Dicionário crítico de educação e tecnologias e de educação a distância, editado por D. Mill (Papirus, Campinas, 2018), p. 139.

[27] D. Mill, em: Dicionário crítico de educação e tecnologias e de educação a distância, editado por D. Mill (Papirus, Campinas, 2018), p. 259.

[28] D. Mill, em: Luzes sobre a Aprendizagem Ativa e Significativa: proposições para práticas pedagógicas na Cultura Digital, editado por D. Mill e G. Santiago, (SEaD, São Carlos, 2021).

[29] M. Ferreira, O.L. Silva Filho, M.A. Moreira, G.B. Franz, K.O. Portugal e D.X.P. Nogueira, Revista Brasileira de Ensino de Física 42, e20200057 (2020).

[30] D. Ausubel, The acquisition and retention of knowledge: a cognitive view (Kluwer Academic Publishers, London, 2000).

[31] J.D. Novak, Uma teoria da educação (Pioneira, São Paulo, 1981)

[32] B.M. Moon, R.R. Hoffman, J.D. Novak e J.J. Canas, Applied Concept mapping: Capturing, analyzing, and organizing knowledge (CRC Press, New York, 2011).

[33] F.M. Gonzales e J.D. Novak, Aprendizaje Significativo Technicas Aplicaciones (Ediciones Pedagogicas, Madrid, 1996).

[34] J.D. Novak, Science Education 86, 548 (2002).

[35] C.T. Machado e A.A. Carvalho, Revista Contexto \& Educação 35, 187 (2020).

[36] H.A. Oliveira Jr., Lógica difusa: aspectos práticos e aplicações (Interciência, Rio de Janeiro, 1999).
[37] R. Tanscheit, em: Simpósio Brasileiro de Automação Inteligente (SBAI) (Bauru, 2003).

[38] B. Wilges, G.P. Mateus, S.M. Nassar e R.C. Bastos, Revista RENOTE - Novas Tecnologias na Educação 8, 1 (2010)

[39] G.M. Tiago, M.P.M.A. Baroni e R. Fonseca, Revemat: Revista Eletrônica de Educação Matemática 9, 87 (2014).

[40] S.B.C. Igliori e P.C. Araújo, em: XII-ENEM Encontros Nacionais de Educação Matemática (São Paulo, 2016).

[41] A.C. Barrantes, R.F. Fonseca e G.M. Tiago, Sistema de Inferência Fuzzy Aplicado na Avaliação Discente (Consistec, São Paulo, 2011). 